\title{
Article \\ Impact of Historical Agrarian Landforms on Soil Water Content Variability at Local Scale in West Carpathian Region, Slovakia
}

\author{
Pavol Kenderessy *(D), Marta Dobrovodská, Barbora Šatalová, Miriam Vlachovičová and Andrej Palaj \\ Institute of Landscape Ecology, Slovak Academy of Sciences, 81499 Bratislava, Slovakia; \\ marta.dobrovodska@savba.sk (M.D.); ba.satalova@gmail.com (B.Š.); miriam.vlachovicova@savba.sk (M.V.); \\ andrej.palaj@savba.sk (A.P.) \\ * Correspondence: pavol.kenderessy@savba.sk
}

Citation: Kenderessy, P. Dobrovodská, M.; Šatalová, B.; Vlachovičová, M.; Palaj, A. Impact of Historical Agrarian Landforms on Soil Water Content Variability at Local Scale in West Carpathian Region, Slovakia. Water 2022, 14, 389. https://doi.org/10.3390/w14030389 Academic Editor: Kyoung Jae Lim Received: 20 November 2021 Accepted: 25 January 2022 Published: 27 January 2022 Publisher's Note: MDPI stays neutral with regard to jurisdictional claims in published maps and institutional affiliations.

Copyright: (C) 2022 by the authors. Licensee MDPI, Basel, Switzerland. This article is an open access article distributed under the terms and conditions of the Creative Commons Attribution (CC BY) license (https:// creativecommons.org/licenses/by/ $4.0 /)$.

\begin{abstract}
The historical agrarian landforms (AL) represent man-made features that alter the hydrological process on cultivated hillslopes. Soil water content (SWC) and its spatial and temporal variability represent an important state indicator for understanding of these processes. In order to assess the differences between individual AL in terms of SWC stability, continuous soil moisture measurements at five different monitoring localities characterized by a specific combination of AL and environmental factors were performed. Temporal SWC stability was evaluated using mean relative difference (MRD) and its standard deviation (SDRD). Differences in mean SWC and MRD values demonstrated the difference between saturated inner part of the AL and external parts such as terraced slopes and mounds, soil depths, and slope positions. In order to analyze the relationship between SWC and environmental variables, the methods of constrained ordination were applied. The most influential factors that regulate SWC variability during the periods of rain were identified as: stone content, sand fraction content, slope orientation, type of agrarian landform, and its orientation against the contour lines. Results also pointed to the fact that different factors predominate among individual localities and, therefore, SWC variability reflects the effect of combination of various environmental factors rather than effect of single parameter. Besides the improved understanding of SWC variability, our results also highlight the importance of AL in regulating the hydrological processes at historical agricultural landscape of the West Carpathian region.
\end{abstract}

Keywords: agrarian landforms; soil water content; temporal stability

\section{Introduction}

Agrarian man-made features such as terraces, mounds, stone walls, etc., have been one of the most visible human alterations to Earth's environments since prehistoric times. They also represent important anthropological characteristics in the landscape and very often are recognized as important part of nation's cultural heritage [1]. Their primary function was to support an agriculture in less favorable environmental conditions and to provide a suitable flat area for cultivation operations. [2]. Aside from their primary function of supporting agricultural productivity, they also provide a variety of additional environmental functions and services [3]. It has been documented that well-maintained agrarian landforms represent important geohazard mitigation and soil conservation measures. By reducing the slope inclination and slope length, they also contribute to the modification of hydrological connectivity and reduction of overland flow and its velocity $[4,5]$. The authors of [6] demonstrated that continuous abandonment of soil water conservation features, such step terraces, resulted in a substantial increase in total drainage area and hydrological connectivity. Similarly, [7] used a modelling approach in order to determine the impact of farmland terraces on runoff connectivity. The results showed a substantial reduction of sediment transport to the outlet channel of a catchment when terraced features were present. The effectiveness of stone bounds and trenches in reducing runoff and soil loss in 
the semi-arid environment was also demonstrated by [8]. Several authors described the effect of overland flow and flow velocity reduction, which promotes the water infiltration in relatively flat areas created between terraces [9-11]. Deeper soils at a flat surface significantly contribute to increase in infiltration rates and sediment transport reduction. The authors of [12] described the effect of well-maintained Mediterranean terraces capable of fully retaining the rainfall of approximately $50 \mathrm{~mm}$ over $24 \mathrm{~h}$ without runoff generation. The retention processes typically mainly affect the inner parts of the terraces, which become saturated during the rainfall periods [13]. In contrast, other studies related to water infiltration on terraces demonstrated that the infiltration capacity of the soil is not particularly affected by terracing; however, the soil stays humid for a longer period in the flat part of the terrace than at the sloping fields [14].

Based on examples from the above mentioned studies, it is evident that man-made agrarian features alter the hydrological processes on steep slopes by changing the slope topography, original soil composition, and land use. Such effects could be studied using suitable indicators, such as soil water content (SWC), which represents a key state indicator for understanding of large number of hydrological processes [15]. Furthermore, another important application of the SWC temporal stability concept is the identification of sample SWC locations and the use of these locations to estimate the mean SWC values [16,17]. Spatial and temporal SWC variability are affected by a number of environmental factors; however, the effects of these multiple factors are rather complex because of their mutual interaction. As a result, a numerous research studies have focused on identifying the mechanisms that influence SWC variability and characterize its link to various environmental factors such as the topography, soil properties, vegetation, climate, seasonality, and scale of the study area [18-21].

Some authors consider topography and soil properties, particularly soil texture, to be the most influential factors. They also confirmed a strong positive relationship between clay content and temporal SWC stability [22-24]. At a local scale, soil moisture is also affected by variables such as bulk density [25], land use [26,27], and geomorphological characteristics such as relative elevation, slope gradient, slope position, slope shape, slope aspect, and soil depth [28-30]. Despite the fact that most of the above-mentioned studies focused on the effect of more than one environmental factor, it still remains challenging to assess the impact of a single factor on a field scale, since mutual interactions of multiple factors has to be considered [31]. According to [32], the combination of climate, soil, topography, and vegetation has a rather more comprehensive impact on SWC stability than a single dominant factor. As a result, reaching an agreement on factors that influence SWC's temporal stability is still challenging.

This study is based on data from continuous SWC measurements taken from April to December 2016 on five different monitoring localities and 60 sampling locations. The main objectives of our study are (1) to evaluate annual SWC variability and its temporal stability at localities with presence of historical agrarian landforms (AL), (2) to identify dominant environmental factors that influence the temporal SWC stability, and (3) to contribute towards an improved understanding of the SWC variability of the historical agrarian landscape of West Carpathians.

\section{Materials and Methods}

The study was carried out at Liptovská Teplička ( $48^{\circ} 57^{\prime} 59^{\prime \prime} \mathrm{N}, 20^{\circ} 5^{\prime} 20^{\prime \prime}$ E, Figure 1), located in the Western Carpathians in the eastern part of the Low Tatras Mts., at altitude between 846-1429 $\mathrm{m}$ a.s.l. The study site represents a specific example of a mountainous landscape, characterized by a well-preserved mosaic of man-made agrarian features, including narrow fields separated by balks, terraces, mounds, and their combinations [33,34]. This region also endured the pressures of agricultural collectivization, typical for socialist agriculture, characterized by intensive management on large-scale field blocks created by ploughing the field borders and merging small agricultural holdings $[35,36]$. The soils are represented by mostly shallow Rendzic Leptosols and Cambisols with a high rock 
fraction content [37]. In addition, anthropogenic man-made features such as productive plots with balks (made of stones collected at productive plots) are covered by Anthropic Regosols. The land-cover in the whole region is dominated by fir and fir-spruce forests, spruce monocultures, and partially also acidophilous beech forests, while in the agrarian part, the land-cover is represented mostly by various types of grasslands and meadows [38].

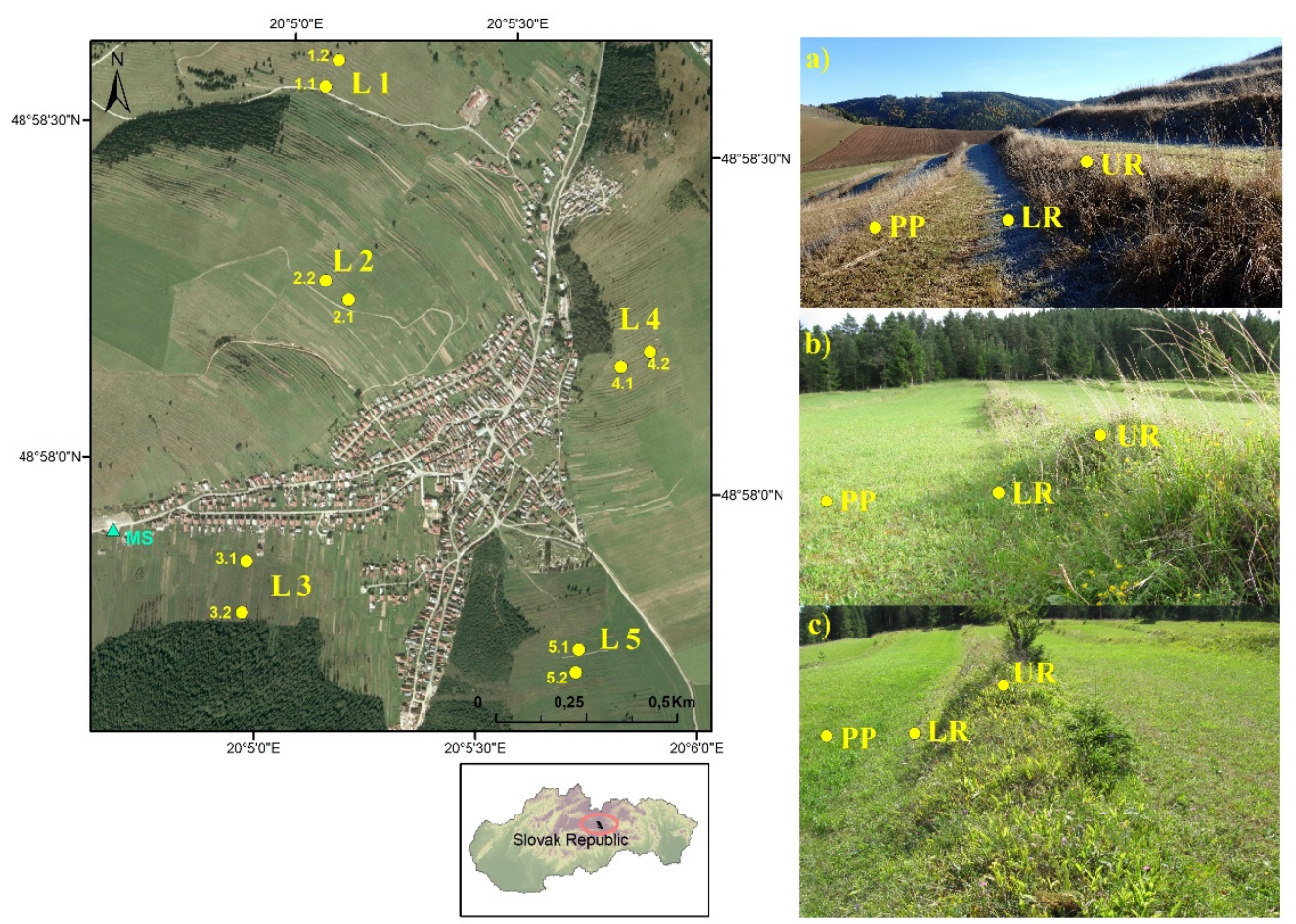

Figure 1. Localization of the study site of Liptovská Teplička village and typology of agrarian landforms (AL): (a) terrace (b) mound (c) mound-terrace; L1-L5-monitoring localities; MS-ground meteorological station.

The specific character and typology of the agrarian region in Liptovská Teplička resulted from long-term historical cultivation process and specific ownership rights related to the Tripartium Law of 1517, valid until 1947. The whole agrarian area was then continuously divided into a number of narrow parcel strips divided by linear landforms such as (1) terraces, (2) mounds, and (3) mound-terraces (Figure 1). During the socialistic agricultural collectivization after 1975, many of the agrarian landforms (AL) were removed and replaced by intensive grasslands and large arable fields. However, a distinctive mosaic of pastures, meadows, small-scale arable fields, and AL has been preserved up to these days, mainly due to exposed natural conditions, which are largely unsuited for regular farming practices. Based on data from study conducted by [39] and updated during the field campaign, the total area of agriculturally utilized land represents $1313 \mathrm{ha}$, out of which 386 ha is characterized as a traditional agrarian landscape. The area with presence of AL covers 293 ha, while $65 \%$ are characterized as terraces and 35\% are represented by mounds and mound-terraces.

\subsection{Rainfall Data Collection and Analysis}

The study site belongs to a cold climatic region with an average temperature varying between 12 and $16{ }^{\circ} \mathrm{C}$ in July and between -6 and $-7^{\circ} \mathrm{C}$ in January. The rainfall data were collected during the year 2016 using automatic ground meteorological station installed in the central part of the study area. According to annual climatological report elaborated by Slovak Hydrometeorological Institute (SHMI) [40], the year 2016 was very rich in atmospheric precipitation, as demonstrated by the high average territorial precipitation totals. In Slovakia, the annual territorial total for 2016 (January-December) reached amount 
of $895 \mathrm{~mm}$. For the hydrological year (November 2015 to October 2016), the total of 890 mm was reached, which represents 120 percent of the 1961-1990 average. In terms of seasonality, the majority of the precipitation fell in the winter of $2015 / 16$, up to $142 \%$ of normal $(200 \mathrm{~mm})$, with February 2016 being the most contributing month $(320 \%$ of normal). October (218\%) and July (191\%) were also among the months with the highest precipitation rates. In contrast, December (57\%), May (65\%), and March (66\%) could be considered as months with the least amount of precipitation (Figure 2). In addition, Figure 2 also shows comparison of values recorded by automatic meteorological station with 30 years monthly regional simulation provided by SHMI.

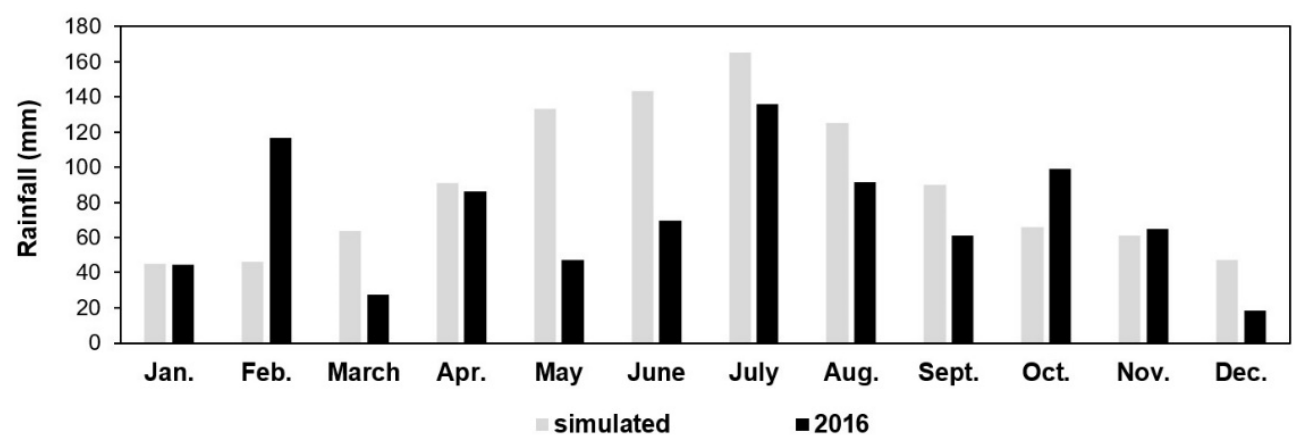

Figure 2. Thirty years of monthly simulated rainfall amounts vs. monthly rainfall rates recorded at the study site in 2016. Source: SHMI, 2021.

An automatic ground meteorological station located in the study area (Figure 1) provided rainfall values $(\mathrm{mm})$ recorded in $10 \mathrm{~min}$ time steps. According to the methodology proposed by [41], the rainfall data were reclassified into hourly precipitation rates, which were used to characterize individual precipitation events. Each precipitation event was separated from the previous one by a minimum $10 \mathrm{~h}$ break. For each event, the total amount (mm), duration (hours), and intensity $\left(\mathrm{mm}\right.$ hour $\left.^{-1}\right)$ were calculated. In total, there were 71 individual precipitation events identified during the monitoring period. The total annual precipitation rate reached a value of $861 \mathrm{~mm}$. The lowest precipitation event was characterized by a total rainfall amount of $0.2 \mathrm{~mm}$ and a duration of $1 \mathrm{~h}$. The highest precipitation rates were recorded in February $(166.6 \mathrm{~mm})$, July $(135.8 \mathrm{~mm})$, August $(91.4 \mathrm{~mm})$, and October $(99 \mathrm{~mm})$, which corresponds to the overall climatological situation described above. The highest rainfall event was recorded on 17 July 2016, with a total amount of $51 \mathrm{~mm}$ and duration of $18 \mathrm{~h}$ (Figure 3).

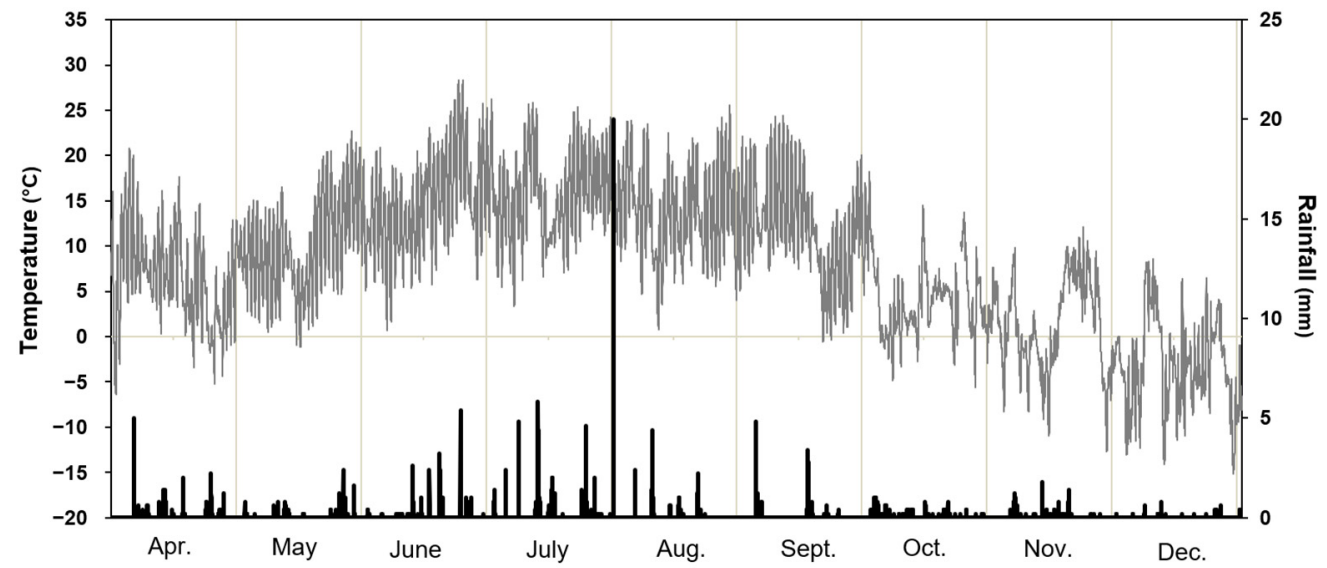

Figure 3. Precipitation and temperature records for the study area of Liptovská Teplička for the monitoring period April-December 2016. 


\subsection{SWC Data Collection and Environmental Variables}

The soil water content (SWC) data were recorded between April and December 2016. Continuous SWC measurements were conducted using EC-5 soil moisture sensors determining the volumetric water content (VWC, $\mathrm{m}^{3} / \mathrm{m}^{3}$ ) attached to EM5b analogue data loggers (Decagon devices, Inc., Pullman, WA, USA). The sensors were installed at a depth of $10 \mathrm{~cm}$ and $30 \mathrm{~cm}$ in the upper soil layer. Due to high rock fraction content, the sensors could be safely installed only to a depth of $30 \mathrm{~cm}$. The monitoring localities (L) were carefully selected in order to capture the representative sample of AL presented in the study area, such as terraces, mounds, and mound-terraces, and diverse natural conditions, land cover, and management (Table 1). In total, five monitoring localities were selected. In order to capture the effect of slope position on SWC variability, at each locality, one footslope (1) and one upslope (2) position were selected. Furthermore, at each slope position, three different positions on agrarian landform were selected-the upper rim (UR) and the lower rim (LR) of the balk, and the productive plot (PP) (Figure 1). In total, there were 60 sampling points selected.

Rock permeability for individual sampling sites was assessed using the database of mean values of hydraulic conductivity $(\mathrm{K})$ set for 156 different aquifer types (31 Quaternary and 125 pre-Quaternary) delineated on the Slovak territory operated by the State Geological Institute of Dionýz Štúr (SGIDŠ). This database was developed using the data from the hydrogeological boreholes and wells maintained by SGIDŠ. For this purpose, 16729 pumping tests were reinterpreted for rock hydraulic properties assessment-hydraulic conductivity $\left(\mathrm{K}, \mathrm{m} \cdot \mathrm{s}^{-1}\right)$ and transmissivity $\left(\mathrm{T}, \mathrm{m}^{2} \cdot \mathrm{s}^{-1}\right)$. Borehole tests reinterpretation for $\mathrm{K}$ and $\mathrm{T}$ values as well as their geometric means were then attributed to individual aquifer types and categorized into eight classes, from slightly permeable to very highly permeable [42]. The whole study area was divided into three classes. Class 1 (high permeability, $\mathrm{k}=2.37 \times 10^{-5}$ ) refers to ramsau gray layered dolomites, Class 2 (medium permeability, $\mathrm{k}=8.49 \times 10^{-6}$ ) refers to gray and dark gray aluminous limestones (sometimes with corneal tubers), silks, siltstones, and aluminous shales, and Class 3 (low permeability, $\mathrm{k}=1.81 \times 10^{-56}$ ) is represented by gray to black cyclically arranged sandstones, shales, and sporadically conglomerates, locally with thin bodies of intermediate volcanics and their volcanoclastics. Geomorphological attributes, such as slope length, slope aspect, altitude, and AL course, were derived using a digital terrain model (DTM) with a resolution of $1 \mathrm{~m}$. The DTM was processed using spatial analyst toolbox and ArcGIS software. Furthermore, at each of the 60 sampling points, disturbed soil samples were collected in order to determine the soil organic matter content, soil texture, stone content, and soil reaction (Table 1). 
Table 1. Characterization of monitoring localities and selected environmental variables.

\begin{tabular}{|c|c|c|c|c|c|c|c|c|c|c|c|c|c|c|c|c|c|c|c|c|c|c|}
\hline \multirow{2}{*}{\multicolumn{2}{|c|}{$\begin{array}{c}\text { Environmental variables } \\
\text { Slope position }\end{array}$}} & & \multicolumn{4}{|c|}{ ML1 } & \multicolumn{4}{|c|}{ ML2 } & \multicolumn{4}{|c|}{ ML3 } & \multicolumn{4}{|c|}{ ML4 } & \multicolumn{4}{|c|}{ ML5 } \\
\hline & & & \multicolumn{2}{|c|}{ (1) } & \multicolumn{2}{|c|}{ (2) } & \multicolumn{2}{|c|}{ (1) } & \multicolumn{2}{|c|}{ (2) } & \multicolumn{2}{|c|}{ (1) } & \multicolumn{2}{|c|}{ (2) } & \multicolumn{2}{|c|}{ (1) } & \multicolumn{2}{|c|}{ (2) } & \multicolumn{2}{|c|}{ (1) } & \multicolumn{2}{|c|}{ (2) } \\
\hline \multicolumn{3}{|c|}{ Slope } & \multicolumn{2}{|c|}{$17-25^{\circ}$} & \multicolumn{2}{|c|}{$12-17^{\circ}$} & \multicolumn{2}{|c|}{$7-12^{\circ}$} & \multicolumn{2}{|c|}{$12-17^{\circ}$} & \multicolumn{2}{|c|}{$7-12^{\circ}$} & \multicolumn{2}{|c|}{$12-17^{\circ}$} & 17 & & & & 12 & & & \\
\hline Slope o & entation & & & & & & & & & & & & & & & & & & $\mathrm{N}$ & & & \\
\hline Rock pe & heability & & & & & & & & mec & & & & & & mec & & me & & his & & & \\
\hline & & & & & & & & & & & & & & & & & & & 7 & & & \\
\hline AL & urse & & & & & & & & diag & & & & & & & & & & co & & & \\
\hline Lan & over & & & & & & & & & & & & & & & & & & tris & & & \\
\hline Managem & on PP/AL & & & & & & & & & & & & & & & & & & 21 & & & \\
\hline Upslope a & above ML & & & & & & & htensi & headow & & & & & & & tensiv & neadow & & & mow & azed & \\
\hline Slope & sition & & & & & & & & & & & & & & & & & & (1 & & & \\
\hline Dep & (cm) & & $0-10$ & 30 & $0-10$ & 30 & $0-10$ & 30 & $0-10$ & 30 & $0-10$ & 30 & $0-10$ & 30 & $0-10$ & 30 & $0-10$ & 30 & $0-10$ & 30 & $0-10$ & 30 \\
\hline & & LR & 6.07 & 6.18 & 6.87 & 7.72 & 6.90 & 7.58 & 7.46 & 7.87 & 7.54 & 7.96 & 7.15 & 7.79 & 7.62 & 7.90 & 7.65 & 7.73 & 7.65 & 7.63 & 7.53 & 7.56 \\
\hline & 2O) & $\begin{array}{l}\text { PP } \\
\text { UR }\end{array}$ & $\begin{array}{l}6.47 \\
5.94\end{array}$ & $\begin{array}{l}6.61 \\
6.09\end{array}$ & $\begin{array}{l}5.75 \\
6.77\end{array}$ & $\begin{array}{l}5.83 \\
7.31\end{array}$ & $\begin{array}{l}6.95 \\
6.66\end{array}$ & $\begin{array}{l}7.42 \\
7.30\end{array}$ & $\begin{array}{l}7.45 \\
6.60\end{array}$ & $\begin{array}{l}7.88 \\
7.12\end{array}$ & $\begin{array}{l}7.60 \\
7.60\end{array}$ & $\begin{array}{l}7.82 \\
7.73\end{array}$ & $\begin{array}{l}7.66 \\
7.54\end{array}$ & $\begin{array}{l}7.97 \\
7.58\end{array}$ & $\begin{array}{l}7.65 \\
7.71\end{array}$ & $\begin{array}{l}7.77 \\
761\end{array}$ & $\begin{array}{l}7.70 \\
7.16\end{array}$ & $\begin{array}{l}7.83 \\
7.57\end{array}$ & $\begin{array}{l}7.50 \\
7.68\end{array}$ & 7.72 & $\begin{array}{l}7.65 \\
7.53\end{array}$ & $\begin{array}{l}7.79 \\
770\end{array}$ \\
\hline & & LR & 1.19 & 0.50 & 2.91 & 0.81 & 2.11 & 0.63 & 2.49 & 1.08 & 1.85 & 0.59 & 1.60 & 0.53 & 3.08 & 1.51 & 2.38 & 1.75 & 2.43 & 1.32 & 2.50 & 1.10 \\
\hline & & PP & 0.98 & 1.12 & 2.14 & 1.48 & 1.98 & 1.66 & 2.21 & 0.76 & 1.94 & 1.50 & 2.29 & 1.79 & 2.60 & 1.53 & 2.71 & 1.62 & 2.88 & 1.91 & 2.54 & 1.46 \\
\hline & $2.00-0.05 \mathrm{~mm}$ & PP & 56 & 60 & 51 & 46 & 47 & 46 & 45 & 46 & 50 & 46 & 55 & 61 & 43 & 48 & 48 & 52 & 44 & 46 & 55 & 48 \\
\hline & & UR & 54 & 54 & 55 & 51 & 43 & 43 & 47 & 38 & 49 & 46 & 62 & 59 & 36 & 36 & 36 & 32 & 50 & 38 & 55 & 51 \\
\hline Texture classes & & LR & 37 & 32 & 39 & 34 & 45 & 53 & 47 & 42 & 36 & 43 & 45 & 44 & 50 & 46 & 48 & 45 & 45 & 38 & 33 & 31 \\
\hline$(\%)$ & $0.05-0.002 \mathrm{~mm}$ & PP & 36 & 34 & 39 & 43 & 46 & 47 & 46 & 45 & 45 & 48 & 40 & 37 & 44 & 39 & 42 & 37 & 46 & 45 & 35 & 37 \\
\hline & & UR & 38 & 38 & 39 & 41 & 51 & 49 & 47 & 53 & 47 & 49 & 35 & 37 & 51 & 50 & 51 & 52 & 43 & 47 & 37 & 38 \\
\hline & & LR & 8 & 11 & 8 & 11 & 7 & 17 & 6 & 6 & 2 & 2 & 5 & 5 & 13 & 12 & 13 & 16 & 9 & 11 & 10 & 9 \\
\hline & $<0.002 \mathrm{~mm}$ & PP & 8 & 6 & 10 & 12 & 7 & 8 & 9 & 9 & 5 & 6 & 4 & 2 & 13 & 12 & 10 & 11 & 10 & 10 & 10 & 15 \\
\hline & & UR & 7 & 8 & 6 & 8 & 7 & 7 & 6 & 9 & 4 & 5 & 3 & 5 & 13 & 15 & 13 & 16 & 8 & 16 & 8 & 11 \\
\hline $\begin{array}{c}\text { Stone content } \\
(\%)\end{array}$ & $2-50 \mathrm{~mm}$ & & 30 & 30 & 40 & 70 & 20 & 35 & 25 & 40 & 30 & 50 & 30 & 60 & 30 & 50 & 35 & 70 & 25 & 35 & 30 & 50 \\
\hline & & $\begin{array}{l}\text { Slope } \\
\text { lines, } \\
\text { mowi } \\
\text { above } \\
\text { Sourc }\end{array}$ & $\begin{array}{l}\text { ion: } \\
\text {-appr } \\
\text { dd re } \\
\text { graz } \\
\text { brov }\end{array}$ & $\begin{array}{l}\text { oot } \\
\text { atel } \\
\text { gra } \\
\text { occa } \\
\text { á (2 }\end{array}$ & $\begin{array}{l}\text { (2)- } \\
\text { ig th } \\
\text { rrass } \\
\text { lly } \\
\text { ield }\end{array}$ & $\begin{array}{l}\text { lope; } \\
\text { lines } \\
\text { s, 2- } \\
\text { d gra } \\
\text { rch. }\end{array}$ & $\begin{array}{l}\text { sition } \\
\text { ont-a } \\
\text { sular } n \\
\text { ands, } 1\end{array}$ & $\begin{array}{l}\text { grari } \\
\text { oxin }\end{array}$ & $\begin{array}{l}\text { landf } \\
\text { ly alc } \\
\text { graz } \\
\text { d-re }\end{array}$ & $\begin{array}{l}\text { UR } \\
\text { he c } \\
\text { gras } \\
\text { rly }\end{array}$ & $\begin{array}{l}\text { oper } r \\
\text { ur lin } \\
\text { ds/or } \\
\text { ved ar }\end{array}$ & $\begin{array}{l}\mathrm{LR}- \\
\mathrm{AL} \\
\text { a ye } \\
\text { graz }\end{array}$ & $\begin{array}{l}\text { ower } \\
\text { e: T- } \\
\text { 3-re } \\
\text { grass }\end{array}$ & $\begin{array}{l}\mathrm{PP}- \\
\mathrm{ce}, \\
\mathrm{cm} \\
\mathrm{ls} \text { W }\end{array}$ & $\begin{array}{l}\text { oduct } \\
\text { mou } \\
\text { ng an } \\
\text { AL, ir }\end{array}$ & $\begin{array}{l}\text { plot; } \\
\text { MT- } \\
\text { razir } \\
\text { sive }\end{array}$ & $\begin{array}{l}\text { cours } \\
\text { oundte } \\
\text { rasslar } \\
\text { adow- }\end{array}$ & $\begin{array}{l}\text { diag } \\
\text { ace; } n \\
\text { s/tw } \\
\text { nten }\end{array}$ & $\begin{array}{l}\text { oproxi } \\
\text { ageme } \\
\text { a year } \\
\text { ly util }\end{array}$ & $\begin{array}{l}\text { tely } \\
\text { on I } \\
\text { rtiliz } \\
\text { ed la }\end{array}$ & $\begin{array}{l}\text { onal } \\
\text { AL: 1- } \\
\text { n; up } \\
\text { olock }\end{array}$ & $\begin{array}{l}\text { contou } \\
\text { regula } \\
\text { pe are } \\
\text { eadow }\end{array}$ \\
\hline
\end{tabular}




\subsection{Annual SWC Variability Assessment}

The relationship between SWC, position on slope (footslope, upslope), position at AL LR, PP, UR), soil depth (10, $30 \mathrm{~cm})$, and season (Q-Q1: April-June, Q2: July-September, Q3: October-December.) was investigated by a general linear model (GLM), processing the means with a factorial ANOVA. Division of the dataset into three seasons reflects the seasonal variability of climate conditions, especially precipitation (Figure 2). Data sets were first tested for normality and homogeneity of variance using a Kolmogorov-Smirnov test. When factors were significant $(p<0.05)$, a post hoc Tukey's test was performed. All analyses were performed using the R statistical software package in RStudio version 1.1.383 (RStudio, Inc., Boston, MA, USA) and software Statistica ${ }^{\circledR}$ (StatSoft Inc., Tulsa, OK, USA).

\subsection{Temporal SWC Stability Assessment}

The temporal SWC stability was determined using values of mean relative difference (MRD) $[43,44]$. Relative difference (RD) is defined as the difference between SWC at location $i$ and time $j$ (SWCij) and the average of these values during the time $j$. The RD was calculated as:

$$
R_{i j}=\frac{S W C i j-\overline{S W C} j}{\overline{S W C} j}
$$

where:

SWCij-SWC at location $\mathrm{i}$ and the sampling time $\mathrm{j}$

$\overline{\mathrm{SWC}} \mathrm{j}-$ mean SWC measured at all sampling sites during the sampling time $\mathrm{j}$.

The mean SWC was computed as follows:

$$
\overline{\mathrm{SWC}} \mathrm{j}=\frac{1}{\mathrm{n}} \sum_{\mathrm{i}=1}^{\mathrm{n}} \mathrm{SWC}_{\mathrm{ij}}
$$

where:

$\mathrm{n}$ - the total number of sampling sites (60 in this study)

The MRD and its standard deviation of relative difference (SDRD) was then calculated as:

$$
\begin{gathered}
\text { MRDi }=\frac{1}{m} \sum_{j=1}^{m} R_{i j} \\
\text { SDRDi }=\sqrt{\frac{1}{m-1} \sum_{j=1}^{m}\left(R D_{i j}-M D_{i}\right)^{2}}
\end{gathered}
$$

where:

$\mathrm{m}$ - the total number of sampling times

The MRD and SDRD values were calculated using hourly SWC records for each of the 60 sampling locations for the whole monitoring period from April 2018 until December 2016 (5831 records in total). When the difference between SWCij and $\overline{\text { SWC }} \mathrm{j}$ is lower, the MRD values are closer to zero. Low MRD values indicate high SWC stability, while low SDRD values correspond to high temporal stability and high SDRD values are associated with low temporal variability [45]. In our case, the MRD values were used to indicate high (positive values) and low (negative values) SWC capacity. In addition, the basic statistical variables (mean, standard deviation, coefficient of variation, minimum and maximum value, and their range) were also calculated for each of the monitoring localities, position on slope (footslope, upslope), positions at agrarian landform (UR, LR, PP), and soil depth $(10,30 \mathrm{~cm})$.

\subsection{Assessment of Relationship between SWC and Environmental Variables}

In order to analyze the relationship between soil moisture and environmental variables, the methods of constrained ordination using R statistical software package Vegan [46] were 
applied. As a species data, mean soil moisture values $\left(\overline{\mathrm{SWC}_{p}}\right)$ from all 60 monitoring sites over 71 periods of rain were used. Mean values were calculated as:

$$
\overline{\mathrm{SWC}_{p}}=\frac{1}{\mathrm{n}} \sum_{\mathrm{i}=1}^{\mathrm{n}} \mathrm{SWC}_{p}
$$

where:

$\mathrm{n}$ - the total number of sampling sites (60) at the sampling time $p$, which represents individual precipitation event.

A detrended correspondence analysis (DCA) of the mean SWC was conducted to determine whether a linear or unimodal approach should be used in the ordinations. Since the length of first DCA axis was lower than 0.5 of average standard deviation (SD), redundancy analysis (RDA) was performed in order to quantify the importance of individual environmental variables. With respect to their relevance, the individual variables were checked for collinearity prior to performing the RDA. Using a Pearson's correlation coefficient, a high association $(>0.80)$ between land-use, rock permeability, and AL course was found. After removing highly correlated variables, seven factors were identified in order to explain SWC variability: slope aspect, AL course, AL type, content of sand, silt and clay fraction, soil rock content, soil depth, and soil reaction.

\section{Results}

3.1. Statistical Characteristics of SWC Variability and Its Relation to Monitoring Locality, Slope Position, Soil Depth, and Position on Agrarian Landform

The basic statistical variables describing the variability of SWC values among monitoring localities, positions on slope, soil depth, and positions on agrarian landforms are listed in Table 2. The main SWC values ranged between 0.206 and $0.257 \mathrm{~m}^{3} / \mathrm{m}^{3}$. The maximum values were reached at locality L2 $\left(0.465 \mathrm{~m}^{3} / \mathrm{m}^{3}\right)$, which could be at the same time considered the locality with the highest mean SWC content $\left(0.275 \mathrm{~m}^{3} / \mathrm{m}^{3}\right)$. The lowest SWC content was recorded at locality L3. The footslope positions exhibit higher mean and MRD values as well lower SD and SDRD values in comparison with upslope positions, which indicates higher SWC content and its stability at footslope positions. Concerning the soil depth, the mean SWC values at $10 \mathrm{~cm}$ are higher than in $30 \mathrm{~cm}$, but at the same time, they also exhibit higher SD and SDRD values, which indicate the lower SWC stability at the upper soil layer. UR positions exhibit lower mean SWC values in comparison with LR and PP positions, which exhibit similar values. UR positions also exhibit lower SD and SDRD values.

Table 2. Statistical characteristics of SWC according to monitoring locality (L1-L5), position on slope (footslope, upslope), position at $\mathrm{AL}$ (UR-upper rim, LR-lower rim, and $\mathrm{PP}$-productive plot) and soil depth (10 and $30 \mathrm{~cm})$.

\begin{tabular}{|c|c|c|c|c|c|c|c|c|c|c|}
\hline $\begin{array}{l}\text { Soil Water Content } \\
\left(\mathrm{SWC} / \mathrm{m}^{3} \cdot \mathrm{m}^{-3}\right)\end{array}$ & Mean & Min & Max & SD & CV (\%) & MRD (\%) & SDRD (\%) & $\underset{(\%)}{\operatorname{Min} \_M R D}$ & $\underset{(\%)}{\operatorname{Max} \text { _MRD }}$ & $\begin{array}{c}\text { Range } \\
(\%)\end{array}$ \\
\hline \multicolumn{11}{|l|}{$\begin{array}{c}\text { Monitoring locality } \\
\text { (ML) }\end{array}$} \\
\hline L1 & 0.206 & 0.063 & 0.421 & 0.062 & 30 & -13.94 & 10.40 & -25.86 & 1.47 & 27.33 \\
\hline $\mathrm{L} 2$ & 0.275 & 0.089 & 0.465 & 0.085 & 31 & 16.51 & 12.18 & -19.54 & 57.83 & 77.36 \\
\hline L3 & 0.208 & 0.034 & 0.414 & 0.067 & 32 & -11.40 & 10.53 & -50.11 & 17.89 & 68.01 \\
\hline L4 & 0.249 & 0.049 & 0.458 & 0.083 & 33 & -0.73 & 13.83 & -30.95 & 36.28 & 67.22 \\
\hline L5 & 0.257 & 0.072 & 0.459 & 0.088 & 34 & 9.56 & 10.42 & -54.76 & 53.28 & 108.04 \\
\hline \multicolumn{11}{|l|}{ Slope position } \\
\hline footslope (1) & 0.250 & 0.072 & 0.459 & 0.075 & 30 & 6.28 & 10.85 & -30.95 & 57.83 & 88.77 \\
\hline upslope (2) & 0.223 & 0.034 & 0.465 & 0.087 & 39 & -6.28 & 13.15 & -54.76 & 56.39 & 111.16 \\
\hline \multicolumn{11}{|l|}{ Soil depth } \\
\hline $10 \mathrm{~cm}$ & 0.241 & 0.034 & 0.465 & 0.086 & 36 & 1.13 & 13.25 & -49.04 & 53.28 & 102.32 \\
\hline $30 \mathrm{~cm}$ & 0.232 & 0.038 & 0.440 & 0.078 & 34 & -1.13 & 10.50 & -54.76 & 57.83 & 112.59 \\
\hline
\end{tabular}


Table 2. Cont.

\begin{tabular}{|c|c|c|c|c|c|c|c|c|c|c|}
\hline $\begin{array}{l}\text { Soil Water Content } \\
\left(\mathrm{SWC} / \mathrm{m}^{3} \cdot \mathrm{m}^{-3}\right)\end{array}$ & Mean & Min & Max & SD & CV (\%) & MRD (\%) & SDRD (\%) & $\underset{(\%)}{\operatorname{Min} \_M R D}$ & $\underset{(\%)}{\operatorname{Max}=M R D}$ & $\underset{(\%)}{\text { Range }}$ \\
\hline \multicolumn{11}{|l|}{ Position at AL } \\
\hline upper rim (UR) & 0.223 & 0.034 & 0.458 & 0.077 & 35 & -5.71 & 10.75 & -50.11 & 34.77 & 84.89 \\
\hline lower rim (LR) & 0.242 & 0.058 & 0.456 & 0.087 & 36 & 2.69 & 13.31 & -54.76 & 57.83 & 112.59 \\
\hline productive plot (PP) & 0.244 & 0.049 & 0.465 & 0.081 & 33 & 3.01 & 11.57 & -30.95 & 50.26 & 81.21 \\
\hline
\end{tabular}

SD—standard deviation; $\mathrm{CV}$-coefficient of variation; MRD—mean relative difference; SDRD—standard deviation of mean relative difference.

The SWC variability related to monitoring locality, season, slope position, position at $\mathrm{AL}$, and soil depth is displayed at Figure 4.
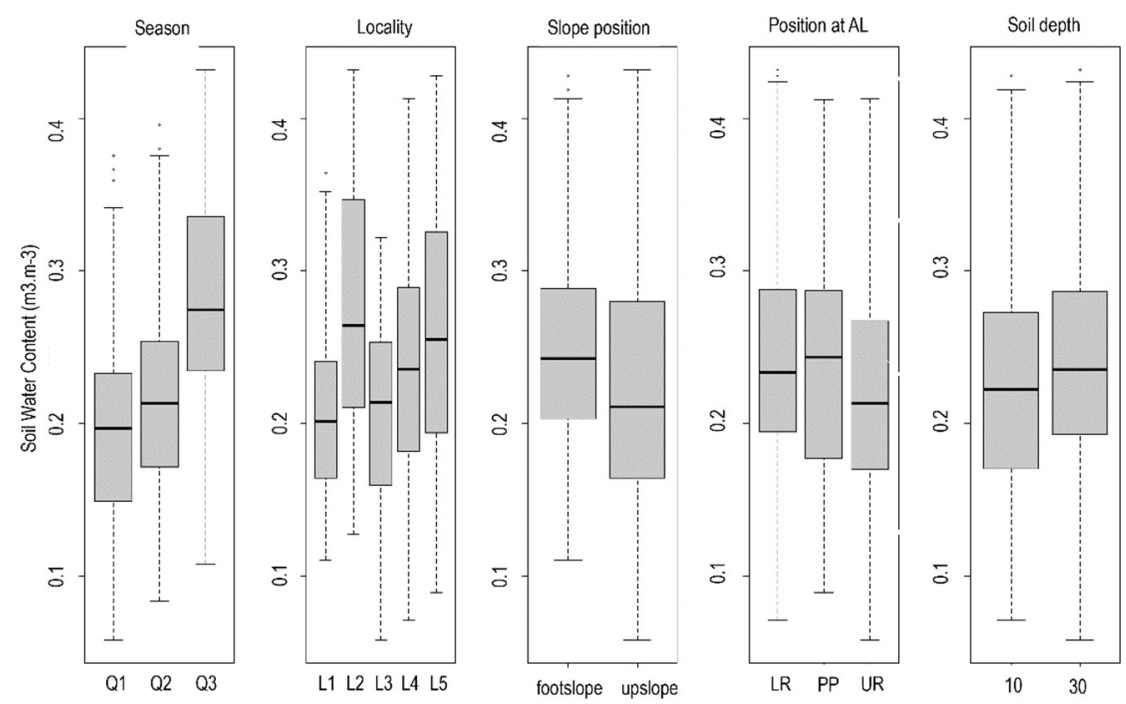

Figure 4. SWC variability related to season (Q-Q1: April-June, Q2: July-September, Q3: OctoberDecember), monitoring locality (L1-L5), position on slope (footslope and upslope), position at agrarian landform (UR-upper rim, LR-lower rim, and PP—productive plot), and soil depth (10 and $30 \mathrm{~cm})$.

As expected, season (Q) had a significant impact on SWC variability $(Q, p<0.001)$. SWC significantly differed between localities $(\mathrm{L}, p<0.001)$. The results of the GLM analysis indicate that SWC significantly depended on slope position (SP, $p<0.001)$. Position at AL and soil depth (D) also reached the significant level of impact (MS, $p<0.001 ; \mathrm{D}, \mathrm{P}=0.007$ ) (Table 3).

Table 3. Results of the general linear model (GLM) analysis.

\begin{tabular}{cccc}
\hline Variables & F & $d f$ & $p$ \\
\hline $\mathrm{D}$ & 7.3 & 1 & $\mathbf{0 . 0 0 7}$ \\
$\mathrm{L}$ & 33.9 & 4 & $<\mathbf{0 . 0 0 1}$ \\
$\mathrm{SP}$ & 37.6 & 1 & $<\mathbf{0 . 0 1}$ \\
$\mathrm{AL}$ & 8.3 & 2 & $<\mathbf{0 0 1}$ \\
$\mathrm{Q}$ & 133.2 & 2 & $<\mathbf{0 0 1}$ \\
$\mathrm{L} \times \mathrm{SP}$ & 32.5 & 4 & $<\mathbf{0 0 1}$ \\
$\mathrm{L} \times \mathrm{AL}$ & 11.3 & 8 & $<\mathbf{0 0 1}$ \\
$\mathrm{SP} \times \mathrm{AL}$ & 18.3 & 2 & $\mathbf{0 . 0 2 2}$ \\
$\mathrm{L} \times \mathrm{Q}$ & 2.3 & 8 & 0.973 \\
$\mathrm{SP} \times \mathrm{Q}$ & 0.0 & 2 & 0.768 \\
$\mathrm{AL} \times \mathrm{Q}$ & 0.5 & 4 & 0.549 \\
$\mathrm{~L} \times \mathrm{SP} \times \mathrm{AL}$ & 0.9 & 8 & 0.297 \\
$\mathrm{~L} \times \mathrm{SP} \times \mathrm{Q}$ & 1.2 & 8 & 1.000 \\
$\mathrm{~L} \times \mathrm{AL} \times \mathrm{Q}$ & 0.2 & 16 & 0.621 \\
$\mathrm{SP} \times \mathrm{AL} \times \mathrm{Q}$ & 0.7 & 4 & 0.999 \\
$\mathrm{~L} \times \mathrm{SP} \times \mathrm{AL} \times \mathrm{Q}$ & 0.3 & 16 & \\
\hline
\end{tabular}

Bold numbers are statistically significant $(p<0,05)$; Q—season; L-monitoring locality (L1-L5); SP—slope position (footslope, upslope), AL—position at agrarian landform (UR - upper rim, LR—lower rim, PP—productive plot), and D—soil depth $(10 \mathrm{~cm}, 30 \mathrm{~cm})$. 


\subsection{Evaluation of SWC Temporal Stability}

The SWC temporal stability was assessed using MRD values, where MRD values were ranked from lowest to highest for each monitoring locality (Figure 5), positions at AL (Figure 6a), position on slope (Figure 6b), and soil depth (Figure 6c). The MRD values show the relative wetness of given sampling locality according to the field average (Table 2). For example, sampling site L3_2_UR_30 exhibits approximately 50\% $( \pm 7 \%)$ lower SWC capacity than the field average; thus, this sampling site could be considered relatively dry. In contrast, sampling site L2_2_PP_10 exhibits approx. 50\% $( \pm 18 \%)$ higher MRD value, which makes it relatively wet in comparison with the field average. When comparing individual localities, it could be observed that locality L1 exhibits significantly lower MRD values than other localities (average MRD $=-13.94 \%$ ) with relatively small difference $(27.33 \%)$ between minimum $(-25.86 \%)$ and maximum $(1.47 \%)$ value. In contrast, locality L2 exhibits the highest MRD with a range from $-19.54 \%$ to $57.83 \%$. With average MRD value equal to $16,51 \%$, this locality could be considered as wettest among the all localities. Lower average MRD value $(-11.40 \%)$, in the case of locality L3, indicates its lower SWC capacity. Locality L4 could be also considered moderately dry with an average MRD value equal to $-0.73 \%$. Locality L 5 is characterized by the highest range $(108,04 \%)$ between minimum $(-54.76 \%)$ and maximum $(53.28 \%)$ value. The highest negative MRD values were reached at sampling sites L5_2_LR_30 $(-54.76 \%)$, L3_2_UR_30 $(-50.11 \%)$, L3_2_UR_10 (-49.04\%), L3_2_LR_10 $(-35.78 \%)$, and L4_1_PP_30 $(-30.95 \%)$, while the highest positive values were recached at sampling sites L2_1_LR_30 (57.82\%), L2_2_LR_30 (56.39\%), L5_1_LR_10 (53.28\%), and L2_2_PP_10 (50.26\%) (Figure 4). When comparing MRD values, depending on the position at AL, it could be observed that PP (3.01\%) and LR $(2.69 \%)$ positions exhibit higher values in comparison with UR positions $(-5.71 \%)$ (Table 2$)$. As indicated by higher SD and SDRD values, the LR positions are considered less stable in comparison with PP and UR. When comparing MRD values at footslope and upslope positions, it could be observed that footslope positions exhibit higher mean MRD (6.28\%) in comparison with upslope positions $(-6.28 \%)$ and, at the same time, footslope positions also exhibit lower SD and SDRD values. Concerning soil depth, the MRD at $10 \mathrm{~cm}$ reached higher mean values $(13.25 \%)$ than in $30 \mathrm{~cm}(10.50 \%)$.

\subsection{The Impact of Selected Environmental Variables}

By constrained ordination, we obtained 10 RDA (constrained) and 49 PCA (unconstrained) axes, while the full model could be evaluated as statistically significant $(\mathrm{F}=3.408 ; p$-value $=0.001)$. The first two RDA axes explain $38 \%$ of total variation, while the first axis is statistically significant $(F=28,086$; $p$-value $=0.004)$. Five factors were identified as highly correlated with average SWC values during the periods of rain. According to the ANOVA-like permutation test for RDA, slope orientation $(F=5.228$; $p$-value $=0.010)$, content of sand fraction $(\mathrm{F}=5.097 ; p$-value $=0.014)$, stone content $(\mathrm{F}=3.968$; $p$-value $=0.034)$, AL type - mound-terrace $(\mathrm{F}=7.164 ; p$-value $=0.004)$, and diagonal AL course $(\mathrm{F}=3.314 ; p$-value $=0.042)$ could be considered the main factors regulating soil moisture variability, while soil depth also has a relatively strong impact $(\mathrm{F}=3.019 ; p$-value $=0.076)$ (Figure 7). Soil reaction and other types and courses of agrarian landforms have a smaller effect during the rain periods. 


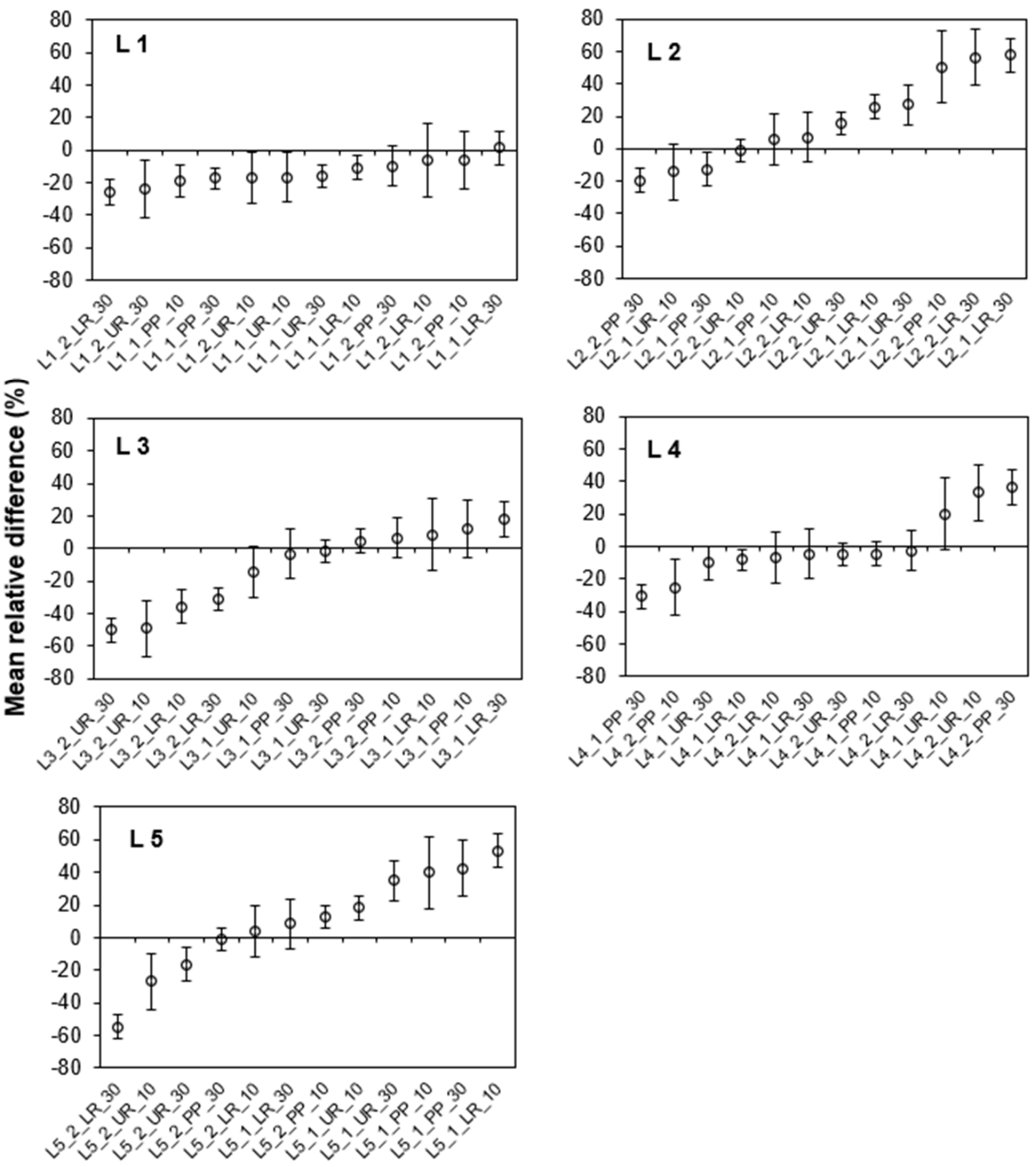

Figure 5. Mean relative difference values (MRD) and its standard deviations (SDRD) calculated for each sampling point, ranked from lowest to highest for each locality (L1-L5). 

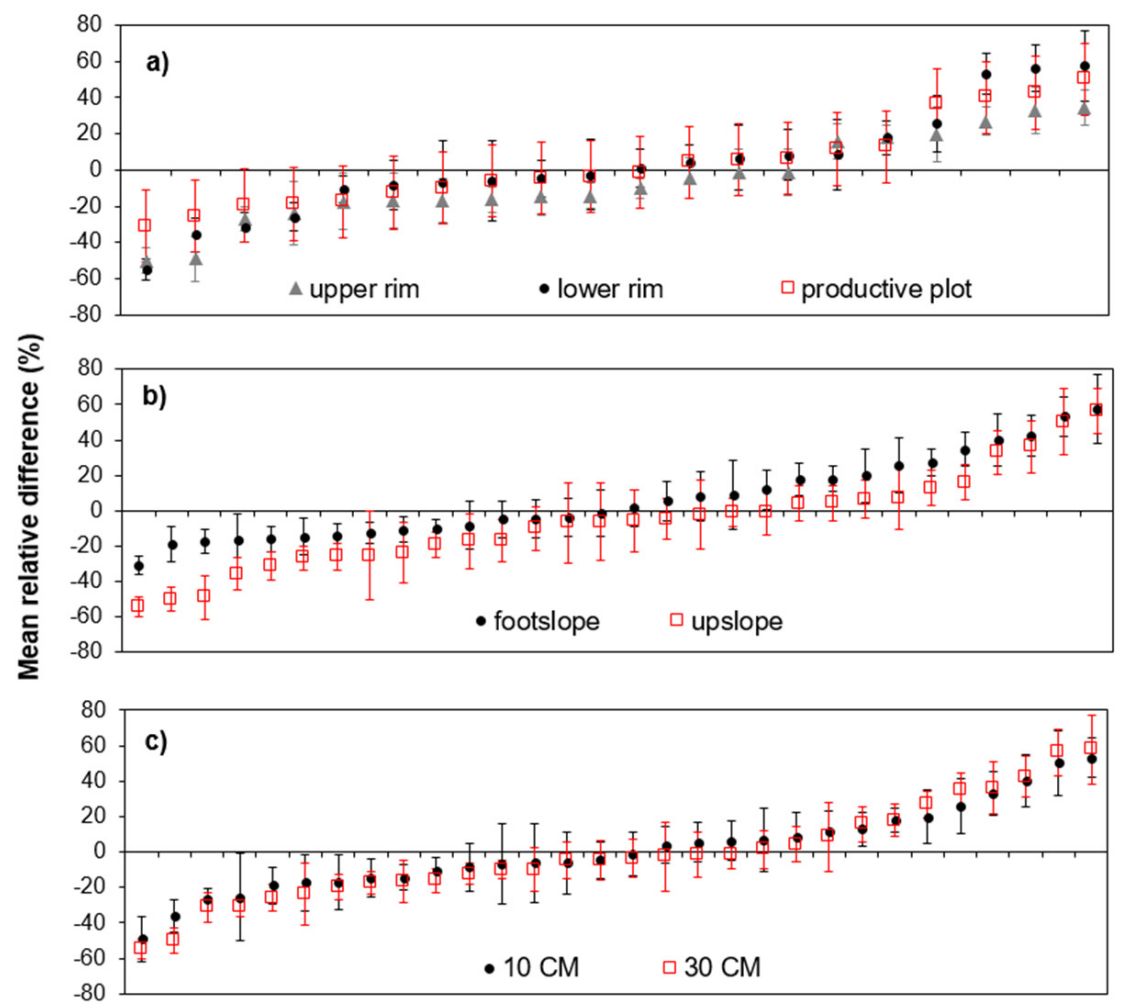

Figure 6. Mean relative difference (MRD) values ranked from lowest to highest according to (a) positions at $\mathrm{AL}$ (UR-upper rim, LR-lower rim, and PP-productive plot); (b) position on slope (footslope and upslope); (c) soil depth (10 and $30 \mathrm{~cm}$ ). Vertical bars represent \pm standard deviation values (SDRD).

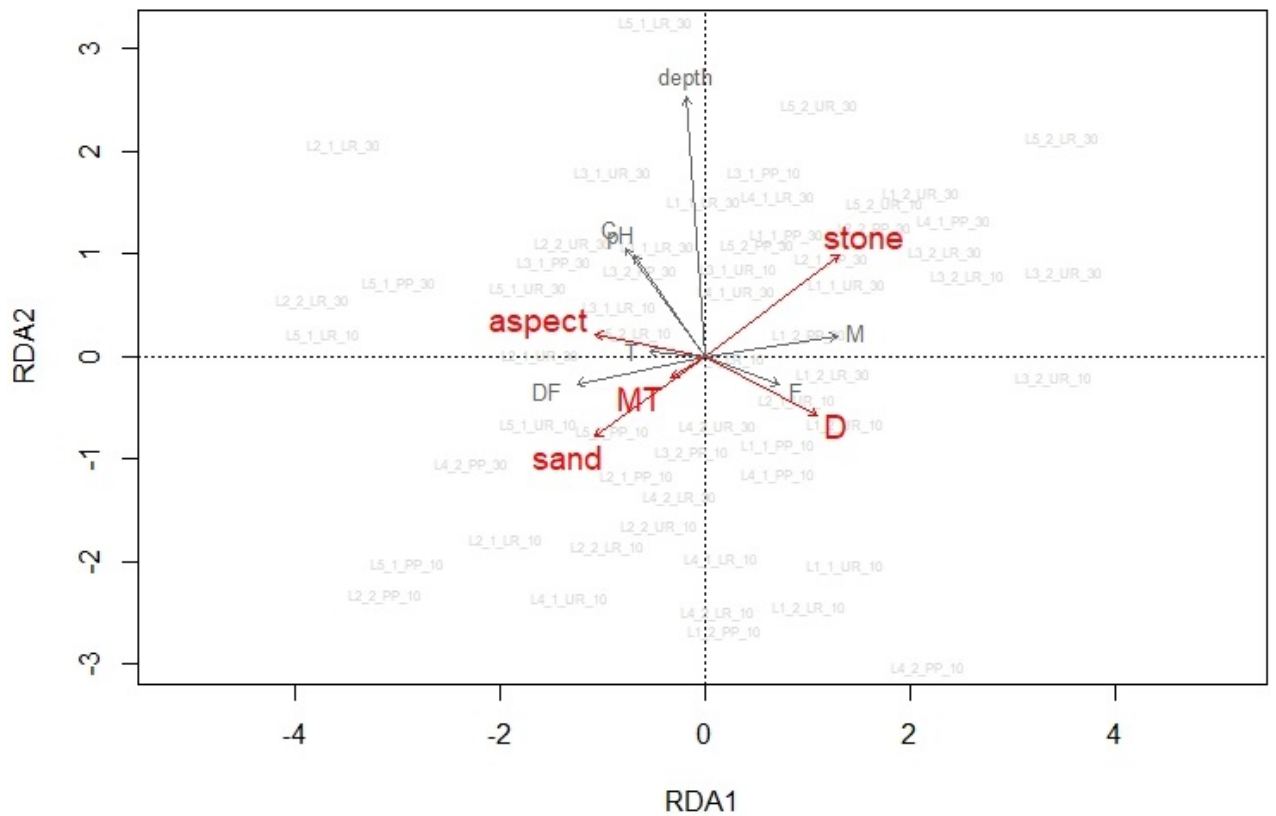

Figure 7. Ordination biplot showing the relationships between average soil moisture over 71 rain periods and environmental variables. Slope orientation, AL course (D-approximately diagonal to contour lines; F-approximately along the fall lines; DF-combination of previous two; C-approximately along the contour lines), AL type (T-terrace; MT—mound-terrace; M-mound), content of sand fraction (sand), stone content (stone), and soil depth and soil reaction $(\mathrm{pH})$ were used to explain the SWC variation. 


\section{Discussion}

\subsection{SWC Temporal Variability and Stability}

Our results clearly demonstrated differences between individual agrarian landforms in terms of SWC spatial and temporal stability. Despite the fact that some localities are similar in terms of their typology, they were characterized by different SWC patterns (Figure 4). Locality L1, which is typologically similar to L2 and L5 (terraces stretched along or diagonal to contour lines), exhibits lower MRD values and their range than other localities. Locality L3, which is similar to L4 (mounds and mound-terraces stretched along the slope line), also exhibits lower MRD values (Table 2). Extensive management at locality L1 (irregular grazing) resulting in biomass accumulation could explain the lower MRD values and their range in comparison with other localities. However, locality L3, which is characterized by intensive management including fertilization, grazing, and regular mowing twice a year, also exhibits lower MRD values in comparison with other localities with similar typology. Numerous studies have demonstrated that vegetation plays an important role in SWC stability [25]. According to [47], the most stable locations were characterized by moderate or above level values of the normalized difference vegetation index. The quality and quantity of aboveground biomass are also altered by land use intensity and management $[20,27]$. Beside the aboveground biomass, the root water absorption has a significant effect on SWC [48]. According to [27], the MRD values were negatively correlated with root density, while SDRD values exhibited positive correlation.

Beside the variability between the individual localities, the SWC values also reflected the differences in relation to slope position, soil depth, and position at AL. As shown in Figure 4, the results of the GLM analysis indicate that SWC significantly depended on slope position (SP, $p<0.001$ ). Position within AL and soil depth also reached the significant level of impact (MS, $p<0.001 ; \mathrm{D}, p=0.007$ ). The higher SD, CV, and SDMR values indicate the lower SWC stability at the upper soil layer (Table 2). Due to the low SWC spatial variability in deeper soil layers, the MRD variation range tends to decrease with depth, regardless of the seasonal fluctuation [49]. Similar to previous research findings, the average SDRD values tend to decline as well with increasing soil depth $[20,50]$. The key explanation for low SDRD values in deeper soil layers was that the effect of environmental factors on deep soil was reduced with depth [32,51], which increased the average SWC content and its temporal stability. However, in the case of our research study, this is only partially valid, since average SWC as well as MRD are lower in $30 \mathrm{~cm}$. This could be explained by the fact, that soils at study area are very shallow, with relatively high stone content.

The differences have been also observed between individual agrarian landforms (LR and UR) and productive plots (PP). Higher average SWC and MRD values at PP positions in comparison with UR positions indicate their higher SWC capacity (Table 2). In contrast, the values of SD and SDRD are lower at UR positions. Despite the fact that LR and PP are relatively similar in terms of average SWC content, the LR positions exhibit higher SD and SDRD values, indicating their lower temporal stability in comparison with PP positions. A similar effect has been also observed by other authors. According to [12,13], the well-preserved terraces in the Mediterranean region could effectively capture and retain the rainfall with an intensity of $50 \mathrm{~mm}$ per $24 \mathrm{~h}$ without generating any excessive runoff. Soils in the inner part of an agrarian landform stay humid for a longer period than at those at steeper field margins [14]. The experiment performed by [52] showed that agrarian landforms, such as stone mounds, exhibit higher infiltration capacity in comparison with the surrounding productive fields. This effect was attributed to higher stone content creating a porous environment and inducing a greater infiltration rate. The above mentioned studies also confirm that agrarian landforms, such as terraces or mounds, not only contribute to reduction and retention of surface flow but at the same time also act as liner drainage elements that affect the hydrophysical properties of the surrounding landscape [53]. This effect could explain the difference between LR or PP and UR positions. The authors of [4] and [54] described the effect of subsurface runoff concentration behind the stone walls when water meets a more impermeable substrate or original soil layer, 
thus creating the hydrological difference between the saturated inner part and external part of agrarian landforms. Higher infiltration rates at relatively dry stone mounds and the creation of subsurface preferential flow could result in higher SD (Table 2) and SDRD (Figure 4) values at LR positions in comparison with PP and LR positions.

\subsection{Factors Influencing the SWC Variability}

The differences in SWC pattern could be explained by the effect various single environmental parameters but also by their combination. The constrained ordination method indicated five major factors (stone content, sand fraction content, slope orientation, AL type, and AL course) regulating SWC variability during the periods of rain (Figure 7). It was assumed that the higher rock content at AL would cause a higher rate of infiltration so that the overall SWC and its stability would be lower, than on the productive plots. The higher rock content may also be related to lower average MRD values at $30 \mathrm{~cm}$ compared to $10 \mathrm{~cm}$ and also lower average MRD values at upslope positions compared to footslope positions. The higher stone content at upslope positions could be associated with the fact that upper slope positions are often a subject of fine soil particles removal due to erosion processes and continuous arable cultivation in the past. The presence of rock fragments can have a great impact on several soil hydrological properties. Stones that are embedded in the soil can alter the soil infiltration rates [55], surface runoff [56,57], or evaporation [58]. An experiment performed by [59] also demonstrated that infiltration rates and hydraulic conductivity values increased with soil rock content exceeding $40 \%$. However, the findings concerning the effect of stones on soil hydrophysical properties are often contradictory. For example, [58] or [60] stated that an increase in infiltration rates and preferential flow is associated with lacunar pores' occurrence (macropores at the stone-soil interface), while other authors reported that the presence of stones can also result in a decrease in hydraulic conductivity [61-63].

The SWC variability was also significantly influenced by soil texture, which also corresponds to findings published by other authors $[50,64]$. Most of the presented studies describe the strong correlation between SWC and clay content; however, in our case, the correlation between clay content and SWC variability was not confirmed. This is mainly due to the fact that soils in the study area are dominated by sand and silt fraction in contrast with clay (Table 1).

Several studies have also indicated a negative correlation between soil moisture and slope gradient [31,65-67], but others also found no significant correlation [68]. A positive correlation between soil moisture and slope aspect also corresponds to the results of previous studies [28,65-67]. Based on the results presented above, we assume that the south orientation beside the biomass accumulation could explain the lower SWC capacity at locality L1 since both factors are specific only to locality L1 (Table 2).

The AL type and its orientation also play an important role in regulating SWC variability on hillslopes. The effect of stone accumulation and creation of flat surface with deeper cultivated soils could result in the creation of a hydrological difference between saturated inner part and external part of agrarian landforms, as described above by [4] and [54]. The AL orientation diagonal to contour lines could have significant impact on SWC variability only in the cases of locality L1 and L2. In addition, in the case of L1, the effect of diagonal AL orientation on SWC variability could be further enhanced by its south orientation. This could explain the low average SWC and MRD rates at L1 in comparison with other localities (Table 2). The presence of mound terraces stretched along the fall lines represent the dominant factor in the case of locality L3. In other localities, an SWC variability could be explained by different factors such as stone content or sand content.

\section{Conclusions}

The main intention of our study was to obtain and analyze experimental data from continued soil moisture measurements in order to demonstrate the impact of various agrarian landforms (AL) and diverse environmental factors on variability and temporal stability 
of soil water content (SWC). Each of the five monitoring localities were characterized by a specific combination of AL and landscape-ecological conditions such as AL type, AL course, slope aspect, soil characteristics, and rock content. Our results revealed several patterns concerning SWC regime and its stability in a traditional agricultural landscape with the presence of AL. The highest SWC variability within monitoring localities was attributed to differences between productive plots and linear elements dividing the productive plots (terraced slopes and mounds). Besides that, the SWC variability was also attributed to slope depth and slope position. We have observed that, on individual monitoring localities, different factors predominate in order to explain the SWC variability and stability. The constrained ordination method indicated five major factors regulating SWC variability during the periods of rain: stone content, sand fraction content, slope orientation, Al type, and its orientation against the contour lines. It is important to mention that all factors may also interact, and thus SWC variability reflects the effect of combination of various environmental factors rather than a distinctive effect of a single parameter. The data in this study demonstrate annual SWC variability instead of just variability during the particular precipitation episodes. Thus, for the future perspective, more attention should be given to assessing the SWC response to different rainfall intensities. Our research also highlights the importance of agrarian landforms in regulating the hydrological processes and supporting the soil water retention on hillslopes.

Author Contributions: Conceptualization, P.K. and M.D.; methodology, P.K. and M.D.; software, M.V. and A.P.; validation, P.K. and B.Š.; formal analysis, P.K.; investigation, P.K., M.D. and B.Š.; resources, P.K.; data curation, P.K. and B.Š; writing-original draft preparation, P.K.; writing-review and editing, P.K.; visualization, P.K.; supervision, P.K.; project administration, M.D.; funding acquisition, M.D. All authors have read and agreed to the published version of the manuscript.

Funding: This research was funded by the Ministry of Education of the Slovak Republic, the Slovak Academy of Sciences with the Grant No. 2/0135/ 22 "Research of specific landscape elements of bio-cultural landscape in Slovakia" and Slovak Research and Development Agency with the Grant No. APVV-17-0377 "Assessment of recent changes and trends in agricultural landscape of Slovakia".

Institutional Review Board Statement: Not applicable.

Informed Consent Statement: Not applicable.

Data Availability Statement: The data presented in this study are available on request from the corresponding author.

Acknowledgments: The authors want to thank the Ministry of Education of the Slovak Republic, the Slovak Academy of Sciences and Slovak Research and Development Agency for supporting this work. The authors also want to thank to Agricultural Cooperative Farm in Liptovská Teplička and local farmer František Ivan for the assistance in setting up the sampling plots.

Conflicts of Interest: The authors declare no conflict of interest.

\section{References}

1. Varotto, M.; Bonardi, L.; Tarolli, P. (Eds.) Introduction. In World Terraced Landscapes: History, Environment, Quality of Life; Springer: Cham, Switzerland, 2019; Volume 9, pp. 1-4. [CrossRef]

2. Tarolli, P.; Preti, F.; Romano, N. Terraced landscapes: From an old best practice to a potential hazard for soil degradation due to land abandonment. Anthropocene 2014, 6, 10-25. [CrossRef]

3. Wei, W.; Chen, D.; Wang, L.; Daryanto, S.; Chen, L.; Yu, Y.; Lu, Y.; Sun, G.; Feng, T. Global synthesis of the classifications, distributions, benefits and issues of terracing. Earth-Sci. Rev. 2016, 159, 388-403. [CrossRef]

4. Arnáez, J.; Lana-Renault, N.; Lasanta, T.; Ruiz, P.; Castroviejo, J. Effects of farming terraces on hydrological and geomorphological processes. A review. Catena 2015, 128, 122-134. [CrossRef]

5. López-Vicente, M.; Poesen, J.; Navas, A.; Gaspar, L. Predicting runoff and sediment connectivity and soil erosion by water for different land use scenarios in the Spanish Pre-Pyrenees. Catena 2013, 102, 62-73. [CrossRef]

6. Agnoletti, M.; Errico, A.; Santoro, A.; Dani, A.; Preti, F. Terraced Landscapes and Hydrogeological Risk. Effects of Land Abandonment in Cinque Terre (Italy) during Severe Rainfall Events. Sustainability 2019, 11, 235. [CrossRef]

7. Lesschen, J.; Schoorl, J.; Cammeraat, E. Modelling runoff and erosion for a semi-arid catchment using a multi-scale approach based on hydrological connectivity. Geomorphology 2009, 109, 174-183. [CrossRef] 
8. Taye, G.; Poesen, J.; Vanmaercke, M.; van Wesemael, B.; Martens, L.; Teka, D.; Nyssen, J.; Deckers, J.; Vanacker, V.; Haregeweyn, N.; et al. Evolution of the effectiveness of stone bunds and trenches in reducing runoff and soil loss in the semi-arid Ethiopian highlands. Zeitschrift für Geomorphologie 2015, 59, 477-493. [CrossRef]

9. van Wesemael, B.; Poesen, J.; Solé-Benet, A.; Barrionuevo, L.C.; Puigdefábregas, J. Collection and storage of runoff from hillslopes in a semi-arid environment: Geomorphic and hydrologic aspects of the aljibe system in Almeria Province, Spain. J. Arid. Environ. 1998, 40, 1-14. [CrossRef]

10. Lü, H.; Zhu, Y.; Skaggs, T.H.; Yu, Z. Comparison of measured and simulated water storage in dryland terraces of the Loess Plateau, China. Agric. Water Manag. 2009, 96, 299-306. [CrossRef]

11. Xu, G.; Zhang, T.; Li, Z.; Li, P.; Cheng, Y.; Cheng, S. Temporal and spatial characteristics of soil water content in diverse soil layers on land terraces of the Loess Plateau, China. Catena 2017, 158, 20-29. [CrossRef]

12. Llorens, P.; Gallart, F. Small basin response in a Mediterranean mountainous abandoned farming area: Research design and preliminary results. Catena 1992, 19, 309-320. [CrossRef]

13. Gallart, F.; Llorens, P.; Latron, J.; Muñoz, D.R. Hydrological processes and their seasonal controls in a small Mediterranean mountain catchment in the Pyrenees. Hydrol. Earth Syst. Sci. 2002, 6, 527-537. [CrossRef]

14. Posthumus, H.; Stroosnijder, L. To terrace or not: The short-term impact of bench terraces on soil properties and crop response in the Peruvian Andes. Environ. Dev. Sustain. 2009, 12, 263-276. [CrossRef]

15. Entin, J.K.; Robock, A.; Vinnikov, K.Y.; Hollinger, S.E.; Liu, S.; Namkhai, A. Temporal and spatial scales of observed soil moisture variations in the extratropics. J. Geophys. Res. Earth Surf. 2000, 105, 11865-11877. [CrossRef]

16. Duan, L.; Huang, M.; Li, Z.; Zhang, Z.; Zhang, L. Estimation of spatial mean soil water storage using temporal stability at the hillslope scale in black locust (Robinia pseudoacacia) stands. Catena 2017, 156, 51-61. [CrossRef]

17. He, Z.-B.; Zhao, M.-M.; Zhu, X.; Du, J.; Chen, L.-F.; Lin, P.-F.; Li, J. Temporal stability of soil water storage in multiple soil layers in high-elevation forests. J. Hydrol. 2019, 569, 532-545. [CrossRef]

18. Korres, W.; Reichenau, T.G.; Fiener, P.; Koyama, C.N.; Bogena, H.R.; Cornelissen, T.; Baatz, R.; Herbst, M.; Diekkrüger, B.; Vereecken, H.; et al. Spatio-temporal soil moisture patterns-A meta-analysis using plot to catchment scale data. J. Hydrol. 2015, 520, 326-341. [CrossRef]

19. Sun, F.; Lü, Y.; Fu, B.; Ma, Z.; Yao, X. Spatial explicit soil moisture analysis: Pattern and its stability at small catchment scale in the loess hilly region of China. Hydrol. Process. 2014, 28, 4091-4109. [CrossRef]

20. Liu, B.; Shao, M. Estimation of soil water storage using temporal stability in four land uses over 10 years on the Loess Plateau, China. J. Hydrol. 2014, 517, 974-984. [CrossRef]

21. Martinez, G.; Pachepsky, Y.A.; Vereecken, H.; Hardelauf, H.; Herbst, M.; Vanderlinden, K. Modeling local control effects on the temporal stability of soil water content. J. Hydrol. 2013, 481, 106-118. [CrossRef]

22. Gao, X.; Wu, P.; Zhao, X.; Shi, Y.; Wang, J. Estimating spatial mean soil water contents of sloping jujube orchards using temporal stability. Agric. Water Manag. 2011, 102, 66-73. [CrossRef]

23. Jacobs, J.M. SMEX02: Field scale variability, time stability and similarity of soil moisture. Remote Sens. Environ. 2004, 92, 436-446. [CrossRef]

24. Mohanty, B.; Skaggs, T. Spatio-temporal evolution and time-stable characteristics of soil moisture within remote sensing footprints with varying soil, slope, and vegetation. Adv. Water Resour. 2001, 24, 1051-1067. [CrossRef]

25. Gómez-Plaza, A.; Martínez-Mena, M.; Albaladejo, J.; Castillo, V.M. Factors regulating spatial distribution of soil water content in small semiarid catchments. J. Hydrol. 2001, 253, 211-226. [CrossRef]

26. Chen, L.; Wang, J.; Wei, W.; Fu, B.; Wu, D. Effects of landscape restoration on soil water storage and water use in the Loess Plateau Region, China. For. Ecol. Manag. 2010, 259, 1291-1298. [CrossRef]

27. Xu, G.; Huang, M.; Li, P.; Li, Z.; Wang, Y. Effects of land use on spatial and temporal distribution of soil moisture within profiles. Environ. Earth Sci. 2021, 80, 128. [CrossRef]

28. Famiglietti, J.; Rudnicki, J.; Rodell, M. Variability in surface moisture content along a hillslope transect: Rattlesnake Hill, Texas. J. Hydrol. 1998, 210, 259-281. [CrossRef]

29. Geroy, I.J.; Gribb, M.M.; Marshall, H.P.; Chandler, D.G.; Benner, S.G.; McNamara, J.P. Aspect influences on soil water retention and storage. Hydrol. Process. 2011, 25, 3836-3842. [CrossRef]

30. Gómez-Plaza, A.; Alvarez-Rogel, J.; Albaladejo, J.; Castillo, V.M. Spatial patterns and temporal stability of soil moisture across a range of scales in a semi-arid environment. Hydrol. Process. 2000, 14, 1261-1277. [CrossRef]

31. Gwak, Y.; Kim, S. Factors affecting soil moisture spatial variability for a humid forest hillslope. Hydrol. Process. 2016, 31, 431-445. [CrossRef]

32. Vanderlinden, K.; Vereecken, H.; Hardelauf, H.; Herbst, M.; Martinez, G.; Cosh, M.H.; Pachepsky, Y.A. Temporal Stability of Soil Water Contents: A Review of Data and Analyses. Vadose Zone J. 2012, 11, 280-288. [CrossRef]

33. Špulerová, J.; Dobrovodská, M.; Lieskovský, J.; Baca, A.; Halabuk, A.; Kohut, F.; Mojses, M.; Kenderessy, P.; Piscová, V.; Barančok, P.; et al. Inventory and classification of historical structures of the agricultural landscape in Slovakia. Ekológia 2011, 30, 157-170. [CrossRef]

34. Dobrovodská, M. Krajinnoekologický výskum historickej pol'nohospodárskej krajiny v obciach Liptovská Teplička, Osturňa a Malá Franková [The development of relations between man and landscape in a historical]. Ekologia 2006, 25, 38-48. (In Slovak) 
35. Bezák, P.; Mitchley, J. Drivers of change in mountain farming in Slovakia: From socialist collectivisation to the Common Agricultural Policy. Reg. Environ. Chang. 2014, 14, 1343-1356. [CrossRef]

36. Lieskovský, J.; Kenderessy, P.; Špulerová, J.; Lieskovský, T.; Koleda, P.; Kienast, F.; Gimmi, U. Factors affecting the persistence of traditional agricultural landscapes in Slovakia during the collectivization of agriculture. Landsc. Ecol. 2014, 29, 867-877. [CrossRef]

37. Balkovič, J.; Bedrna, Z.; Čurlík, J.; Dlapa, P.; Fulajtár, E.; Gömöryová, E.; Gregor, J.; Hanes, J.; Houšková, B.; Hutár, V.; et al. Morfogenetický Klasifikačný Systém pôd Slovenska. Bazálna Referenčná Taxonómia [Morfogenetic Classification System of Soils in Slovakia]; SPS, NPPC VÚPOP: Bratislava, Slovakia, 2000; 96p. (In Slovak)

38. Ružičková, H.; Dobrovodská, M.; Valachovič, M. Landscape-ecological evaluation of vegetation in relation to the forms of anthropogenic relief in the cadastre of the Liptovská Teplička Village, the Nízke Tatry Mts. Ekológia 1999, 18, 381-400.

39. Špulerová, J.; Dobrovodská, M.; Bezák, P.; Barančok, P.; Boltižiar, M.; David, S.; Dramstad, W.; Ďugová, O.; Fjellstad, W.; Gajdoš, P.; et al. Stratégia ochrany a manažmentu historických štruktúr pol'nohospodárskej krajiny v modelovom území Liptovská Teplička [Strategy of protection and management of historical agrarian structures in Liptovská Teplička village]; Institute of landscape Ecology SAS: Bratislava, Slovak, 2011; 152p. (In Slovak)

40. Pecho, J.; Ivaňáková, G.; Kajaba, P.; Labudová, L.; Št’astný, P.; Turňa, M. Climatological assessment of the year 2016 in Slovakia. Meterol. J. 2017, 20, 23-27. (In Slovak)

41. Šamaj, F.; Valovič, S. Intensities of short-term rainfall in Slovakia. In Proceedings of Works of SHMI; SPN: Bratislava, Slovakia, 1973; Nr. 5. (In Slovak)

42. Malík, P.; Švasta, J.; Bottlik, F. Hydrogeological Boreholes and Wells Database and its Use on Regional Rock Permeability Determination. Slovak Geol. Mag. 2016, 16, 67-93.

43. Vachaud, G.; De Silans, A.P.; Balabanis, P.; Vauclin, M. Temporal Stability of Spatially Measured Soil Water Probability Density Function. Soil Sci. Soc. Am. J. 1985, 49, 822-828. [CrossRef]

44. Ben-Salem, N.; Álvarez, S.; López-Vicente, M. Soil and Water Conservation in Rainfed Vineyards with Common Sainfoin and Spontaneous Vegetation under Different Ground Conditions. Water 2018, 10, 1058. [CrossRef]

45. López-Vicente, M.; Álvarez, S. Stability and patterns of topsoil water content in rainfed vineyards, olive groves, and cereal fields under different soil and tillage conditions. Agric. Water Manag. 2018, 201, 167-176. [CrossRef]

46. Oksanen, J.; Blanchet, F.G.; Friendly, M.; Kindt, R.; Legendre, P.; McGlinn, D.; Minchin, P.R.; O’Hara, R.B.; Simpson, G.L.; Solymos, P.; et al. Vegan: Community Ecology Package. R Package Version 2.5-7. 2020. Available online: https://CRAN.R-project.org/ package $=$ vegan $($ accessed on 15 November 2021).

47. Jacobs, J.M.; Hsu, E.-C.; Choi, M. Time stability and variability of Electronically Scanned Thinned Array Radiometer soil moisture during Southern Great Plains hydrology experiments. Hydrol. Process. 2010, 24, 2807-2819. [CrossRef]

48. Graefe, S.; Leuschner, C.; Coners, H.; Hertel, D. Root functioning in tropical high-elevation forests: Environmental vs. biological control of root water absorption. Environ. Exp. Bot. 2011, 71, 329-336. [CrossRef]

49. Xu, M.; Xu, G.; Cheng, Y.; Min, Z.; Li, P.; Zhao, B.; Shi, P.; Xiao, L. Soil Moisture Estimation and Its Influencing Factors Based on Temporal Stability on a Semiarid Sloped Forestland. Front. Earth Sci. 2021, 9, 52. [CrossRef]

50. Penna, D.; Brocca, L.; Borga, M.; Fontana, G.D. Soil moisture temporal stability at different depths on two alpine hillslopes during wet and dry periods. J. Hydrol. 2013, 477, 55-71. [CrossRef]

51. Gao, L.; Lv, Y.; Wang, D.; Muhammad, T.; Biswas, A.; Peng, X. Soil water storage prediction at high space-time resolution along an agricultural hillslope. Agric. Water Manag. 2016, 165, 122-130. [CrossRef]

52. Štibinger, J. Infiltrační schopnosti agrárních valů [Infiltration Capabilities of Agrarian Land Mounds]. Stavební Obzor $2011,2,6$.

53. Kovár̆, P.; Štibinger, J.; Kasl, M. Agrární valy a vodní režim [Agrarian Mounds and their Water Regime]; The Czech University of Life Sciences in Prague: Prague, Czech Republic, 2011; 20p.

54. Preti, F.; Guastini, E.; Penna, D.; Dani, A.; Cassiani, G.; Boaga, J.; Deiana, R.; Romano, N.; Nasta, P.; Palladino, M.; et al Conceptualization of Water Flow Pathways in Agricultural Terraced Landscapes. Land Degrad. Dev. 2018, 29, 651-662. [CrossRef]

55. Brakensiek, D.; Rawls, W. Soil containing rock fragments: Effects on infiltration. Catena 1994, 23, 99-110. [CrossRef]

56. Sauer, T.J.; Logsdon, S.D. Hydraulic and Physical Properties of Stony Soils in a Small Watershed. Soil Sci. Soc. Am. J. 2002, 66, 1947-1956. [CrossRef]

57. Poesen, J.; Lavee, H. Rock fragments in top soils: Significance and processes. Catena 1994, 23, 1-28. [CrossRef]

58. Beckers, E.; Pichault, M.; Pansak, W.; Degré, A.; Garré, S. Characterization of stony soils' hydraulic conductivity using laboratory and numerical experiments. SOIL 2016, 2, 421-431. [CrossRef]

59. Beibei, Z.; Ming'An, S.; Hongbo, S. Effects of rock fragments on water movement and solute transport in a Loess Plateau soil. Comptes Rendus Geosci. 2009, 341, 462-472. [CrossRef]

60. Fiès, J.C.; Louvigny, N.D.E.; Chanzy, A. The role of stones in soil water retention. Eur. J. Soil Sci. 2002, 53, 95-104. [CrossRef]

61. Ma, D.; Shao, M. Simulating infiltration into stony soils with a dual-porosity model. Eur. J. Soil Sci. 2008, 59, 950-959. [CrossRef]

62. Ma, D.; Shao, M.; Zhang, J.; Wang, Q. Validation of an analytical method for determining soil hydraulic properties of stony soils using experimental data. Geoderma 2010, 159, 262-269. [CrossRef]

63. Novák, V.; Kňava, K.; Šimůnek, J. Determining the influence of stones on hydraulic conductivity of saturated soils using numerical method. Geoderma 2011, 161, 177-181. [CrossRef] 
64. Chaney, N.W.; Roundy, J.K.; Herrera-Estrada, J.E.; Wood, E.F. High-resolution modeling of the spatial heterogeneity of soil moisture: Applications in network design. Water Resour. Res. 2015, 51, 619-638. [CrossRef]

65. Kim, S.; Lee, H.; Woo, N.C.; Kim, J. Soil moisture monitoring on a steep hillside. Hydrol. Process. 2007, 21, 2910-2922. [CrossRef]

66. Moore, I.D.; Burch, G.J.; Mackenzie, D.H. Topographic effects on the distribution of surface soil water and the location of ephemeral gullies. Trans. ASAE 1988, 31, 1098-1107. [CrossRef]

67. Western, A.W.; Grayson, R.B.; Bloschl, G.; Willgoose, G.R.; McMahon, T.A. Observed spatial organization of soil moisture and its relation to terrain indices. Water Resour. Res. 1999, 35, 797-810. [CrossRef]

68. Ladson, A.; Moore, I. Soil water prediction on the Konza Prairie by microwave remote sensing and topographic attributes. J. Hydrol. 1992, 138, 385-407. [CrossRef] 